\title{
Necrotizing Pancreatitis: Update and When to Operate
}

\author{
Ricardo Antonio Refinetti, Rodrigo Martinez*
}

Department of Surgery, Federal University in Rio de janeiro, Rio de janeiro, RJ, Brazil

\begin{abstract}
About $20 \%$ of the cases of acute pancreatitis present in a severe form, known as necrotizing pancreatitis. Although this entity was first described centuries ago, many questions about it remain open and as a consequence, many studies are currently published addressing this subject nowadays. We performed a throughout research in the literature, with special attention to the articles published in the last five years and indexed to the PubMed. The following headings were used: Pancreatitis, Surgical procedures, Necrosis. The initial research rendered about 1800 articles, and the ones published in the last five years were evaluated. Some older, but remarkable articles were also included given their importance to this matter. As we further describe, treatment of acute pancreatitis involves a great number of questions, among which the most important are the ones related to the terminology of pancreatic collections, prognostic markers, use of antibiotics, type of diet employed and the approach to infected necrosis. Many radical changes could be seen over the last years on all these topics and a constant updating must be necessarily done by the ones involved in the treatment of this disease.
\end{abstract}

Keywords Pancreatitis, Surgical procedures, Necrosis

\section{Introduction}

Acute pancreatitis (AP) is a disease with an extremely variable clinical spectrum, ranging from a mild disease characterized by minimal abdominal pain, without major systemic repercussions to a much more florid scenario, marked majorly by multiple organ dysfunction and death [1].

The advances observed over the latest years in the knowledge and treatment of this disease, with a great number of clinical and experimental papers published, allowed a better understanding of its evolution as well as an improvement in the results in the treatment of this disease. Despite all that, the physiopathology of acute pancreatitis and its complications remain with many obscure points, making the development of successful therapeutic options a major challenge[1].

AP is an inflammatory disease of the pancreas, associated with many different conditions, known as the etiological agents of PA. Among them, biliary lithiasis and alcohol ingestion are responsible for $80 \%$ of all the cases, with a slight variation according to the population studied[2]. Additionally to these major etiological agents, drugs such as Azatioprin, Sulfonamides, Pentamidine and Valproic Acid with an idiosyncratic behaviour, high doses of thiazides and oestrogens, due to a heightening of triglyceride levels, infections, hypertriglicidemia, hipercalcemia, trauma and

* Corresponding author:

rodrigomartinez@hucff.ufrj.br (Rodrigo Martinez)

Published online at http://journal.sapub.org/cmd

Copyright (C) 2012 Scientific \& Academic Publishing. All Rights Reserved hypotension, such as that seen during cardiac surgery and post-partum, can also cause AP. It should also be stressed that idiopathic cases account for about $10 \%$ of all the cases[1].

Given the huge advancements observed in this field over the last five years, we were encouraged to perform this review focusing the articles published in the last five years and indexed to the PubMed. The following headings were used: Pancreatitis, Surgical procedures, Necrosis. The initial research rendered about 1800 articles and the ones published during the later five years were evaluated. From these articles, we focused mostly on the ones that provided the greatest body of scientific evidences, with a special attention to meta-analyses and randomized prospective studies. Some older, but remarkable, articles were also included given their importance to this matter.

\section{Terminology}

Among the many forms of presentation of AP, the one that certainly represents the major therapeutic challenge is Necro-hemorrhagic pancreatitis (NHAP). Although this entity has already been described for the first time in 1652, its devastating clinical presentation and the frustrating therapeutic results presented up to this moment make it one of the most studied diseases nowadays[3]. In this initial definition, we are already facing a major problem, since the terminology adopted by each author is amazingly variable. This fact makes the comparison among results obtained by different groups and the acceptance of general treatment guidelines troublesome. NHAP accounts for roughly 15 to $20 \%$ of AP cases, with a growing incidence, not only be- 
cause AP is becoming a more frequent disease, but also due to the improvement in life support of those patients suffering from this condition, and a better diagnosis evaluation[4].

The clinical scenario of NHAP can be divided into two major phases: In the first one, which generally comprises the first week of the disease, there is an extensive systemic inflammatory response syndrome (SIRS), and mortality is at this moment mostly related to the general clinical conditions of the patient, so that major therapeutic challenges are profoundly linked to the same ones related to other clinical conditions, such as severe septic shock, in which the same group of initial pathophysiological events can be observed. Later, after the second or third weeks, multi-organic dysfunction is mostly secondary to an incontrollable infected pancreatic necrosis[5].

Coming back to terminology, various classifying systems have historically been used, giving origin to a very wide and not infrequently confusing terminology (Sarles 1965, Marseilhe I, Singer 1984, Marseilhe II, Cambridge 1985). With the objective of ending this problem, Atlanta's classification, proposed in 1992, is composed of the basic elements listed below[6, 7]:

1 - Mild pancreatitis, characterized generally by a benign clinical presentation, and no need for pancreatic parenchyma manipulation.

2 - Severe pancreatitis (SAP), defined as every case of AP associated to organ dysfunction and/or local complications. The local complications are:

A)Liquid collections - defined as those formed just after the beginning of the pathologic process, no true capsule surrounding the collection is seen.

B) Pseudocysts - These are the final evolution of liquid collections that are not fully reabsorbed, since after some weeks there is a trend for a pseudo capsule to be formed, which is truly originated from the surrounding organs.

C)Necrosis - This lesion originates from the self-digestion of pancreatic parenchyma and its surrounding fatty tissue.

Despite being revised by the Acute Pancreatitis Working Group, which included new terms such as "acute peripancreatic fluid collections", in order to optimize the description of the morphological changes in the peripancreatic area, the Atlanta classification is still the most widely accepted in order to describe the different lesions related to $\operatorname{AP}[8,9]$.

Pancreatic pseudocysts and necrosis can become sites of secondary bacterial colonization and infection. Infected pseudocyst is a terminology not present in the Atlanta classification, being otherwise described as a pancreatic abscess. The same pathological entity can be the result of an infected pancreatic necrosis that has suffered a liquefaction process. In all these cases, it is believed that the major mechanism of infection is secondary to bacterial translocation from the intestine, favoured by an increase in intestinal permeability due to the neighbouring inflammatory process. In accordance with that, the major infectious agents are Gram-ne gative enteric bacteria, such as Escherichia coli and Klebisiella spp. Enterococcus species also account to an important percentage of the cases[10]. The widespread use of prophylactic antibiotics, a theme to be better approached elsewhere in this paper, is promoting an increase in the number of infections caused by multi-resistant bacteria and even by fungal agents[11, 12].

\section{Diagnosis of Severe AP and of NHAP}

The first effort to be made, in order to achieve a better treatment of severe AP is to identify those suffering from this entity. In order to accomplish that, many different classifications specific or not to AP can be used, such as Ranson's, Glasgow and APACHE II (Acute Physiology and Chronic Health Care Evaluation II). The presence of three or more of Ranson's criteria or an APACHE II score of more than 8 indicate a case of SAP[6]. According to this, patients presenting high scores must be closely followed up, ideally at the intensive care unit[13]. Severity evaluation can also be accomplished by diagnostic imaging, such as the Balthazar criteria, after the evaluation of the images from a contrast-enhanced computerized tomography (CT). In this scenario, findings of non-perfused areas after at least 24 to 48 hours from the initial symptoms is perhaps one of the most valuable, indicating the presence of pancreatic necrosis, a condition whose extension is directly related to the severity of organ dysfunction and to the risk of infection development[14].

Also valuable in predicting the severity of AP are some molecular markers such as $\mathrm{C}$ reactive protein, interleukin 1 and 6 , tumour necrosis factor- $\alpha$ and some computer-based tools, but the actual use of them is limited, since most of them are not commercially available or not routinely measured[15]. The current literature displays a huge number of articles pointing to new potential prognostic markers for outcome of acute pancreatitis, but a recent review of these papers, despite detecting 184 studies developed with this objective, suggests that the reporting on this type of research is suboptimal, not providing enough information to allow the scientific community to come to solid conclusions about their real value[16]. As a consequence, the great majority of these new markers are not incorporated into clinical practice.

\section{Antibiotic Therapy in SAP}

During the evaluation of patients with SAP, finding necrosis is certainly one of the most alarming aspects. Besides its already described correlation with multiple organ dysfunctions, pancreatic necrosis can also become infected in a great proportion of the cases, especially in the most extensive ones. When necrosis involves more than $50 \%$ of the pancreas, the rate of infection is higher than $40 \%[17]$. Aggravating this scenario, the distinction between infected and 
non-infected necrosis is a difficult task, since virtually every patient with extensive pancreatic necrosis, whether sterile or infected, has a severe clinical presentation, commonly associated with fever, leucocytosis and even SIRS. In these patients, even when sepsis is diagnosed, the source of infection can be widespread, given their poor state of health and the presence of many other risk factors, such as vascular ports, mechanical ventilation and urinary catheters. These factors encouraged many authorities involved on the treatment of SAP to recommend the use of wide spectrum prophylactic antibiotics in individuals with extensive pancreatic necrosis[7]. This is still a much debated issue, given the fact that a great number of papers, with remarkable heterogeneities in experimental designs and clinical groups addressing this question have been published so far. More notably, a group of recently published meta-analyses have failed to show a convincing role for prophylactic antibiotics in these patients, and since then, most groups have moved towards abolishing this practice[18, 19, 20, 21]. Alternatively, the use of non-absorbable antibiotics via the oral route (selective digestive decontamination) as a strategy to reduce the rates of pancreatic necrosis has been recently proposed with some promising results[22, 23]. As a conclusion, although this is still a theme for debate[1], we can assume that currently the most accepted protocol is to initiate the use of antibiotics only when infected pancreatic necrosis is diagnosed, and this could be definitively determined by a positive culture of the material obtained by invasive methods, such as CT-guided fine needle aspiration[24]. An abdominal CT scan demonstrating the presence of gas in or near the necrosis is also considered diagnosis of infection. Alternatively, serum biochemical markers, such as $\mathrm{C}$ reactive protein and mieloperoperoxidase have been tested as predictors of infected pancreatic necrosis, but from the studies published so far, no definitive conclusions about these markers can be taken[25, 26]. A patient with deteriorating clinical/haematological condition with positive blood cultures for enteric bacteria or with serological tests positive for endotoxins could also be a strong candidate for this diagnosis. More recently, a huge prospective paper has shown that clinical and laboratorial parameters such as leucocytosis and high $\mathrm{C}$ reactive levels used in conjunction could be accurate in identifying patients with infected pancreatic necrosis[27]. Adding more complexity to this issue is the fact that, although fine needle aspiration is considered the gold-standard method of diagnosis for infected pancreatic necrosis, with advantages such as the accuracy of $89-100 \%[28]$, and the possibility of guiding the antibiotic therapy[11], it is not widely available and also not free of risks, with the concrete possibility of causing, among other complications, the iatrogenic contamination of the necrosis[29].

\section{Dietetic Management in SAP}

The management of diet in SAP has dramatically changed over the last decades. The importance of returning enteral diet, as a mechanism for inhibiting bacterial translocation and thus reducing the rates of pancreatic infection has been confirmed by a number of papers, which also describe other benefits of this practice, such as reduced hospital stay and fewer surgical interventions, when patients are given diet through a nasoenteral tube positioned at the jejunum[30, 31]. These results challenge the primitive concept that enteral nutrition could stimulate pancreatic secretion and in this manner, aggravate SAP. Some questions do still remain open, concerning issues about the most beneficial diet to be given and the possibility of having the diet delivered into the stomach. The moment to initiate the diet should be personalized, according to the patient's evolution. Of note, Al Samaree and cols. performed a recent systematic review of the literature and concluded that there is level 1 evidence strongly supporting early nutrition through the enteral route in SAP. When dealing with other issues, however, evidence is not so strong. For example, the same authors have found level 2 evidence for using the nasogastric route instead of the nasoenteral and for the use of immune-nutrition[32]. Regarding probiotic use, the same systematic review and two recent meta-analysis describe the lack of beneficial effects of this agents in $\operatorname{SAP}[33,34]$.

\section{Role of Endoscopic Methods in SAP}

A major issue in biliary AP, is the approach to choledocholithiasis, since this could not only be an initiating but also aggravating and perpetuating element. In regard to this, it is believed that in most cases, the common duct stone is no longer present after the clinical syndrome has been established[35]. At the same time, the indiscriminate performance of an endoscopic retrograde cholangiopancreatography (ERCP) can carry a great number of negative results and presents the risk of devastating complications, especially for the patients suffering from SAP. Taking this into consideration, many papers have been published proposing the selective use of ERCP in the early phases of biliary SAP with conflicting results, regarding the real benefit obtained by this therapeutic modality[36, 37, 38, 39]. It should also be considered in this issue the fact that many alternative diagnosis tests have already been developed for the diagnosis of choledocholithiasis, such as ERCP and endoscopic ultrasound, with similar accuracy and much less invasiveness. Taking this into account, most authorities will initially investigate the presence of choledocholithiasis in patients with biliary SAP using less invasive methods and use ERCP whenever there is a confirmation of this diagnosis or when there is associated severe cholangitis or jaundice.

Also noteworthy regarding this issue is the growing use of endoscopic methods for the drainage and even debride- 
ment of pancreatic necrosis, as it will be better described in the next sessions[40].

\section{Role of Surgery and Other Invasive Methods in SAP}

AP is in most cases a non-operatively managed disease[2], but surgery still plays a definite role in a minor number of cases, such as when there is a diagnosis uncertainty and the patient displays signs and symptoms favouring a surgical abdomen. This scenario is becoming increasingly more uncommon with the improvement and widespread use of diagnosis methods such as the abdominal CT scan. More acceptable surgical indications are the situations in which SAP is complicated by entero-mesenteric infarction or abdominal compartment syndrome. In all these cases, surgery is performed at an early stage of the disease, and this is generally followed by an unfavourable clinical course, due to factors such as the aggravation of the systemic inflammatory response and the potential for iatrogenic contamination of the abdominal cavity.

At a later stage of the disease, surgical intervention can be indicated in the management of SAP complicated with an infected necrosis. As it was previously stated, patients presenting SAP should be submitted to a contrast-enhanced abdominal CT scan. This exam is more accurate when performed after 7 days the disease has started, when the necrotic tissue is more easily identified. In such a scenario, most patients will be presenting a sterile necrosis and the probability of infection is directly related to its extension. When there is a suspicion that the necrosis is infected, the gold standard diagnosis method is fine needle aspiration of the necrosis, followed by culture of the aspirated material. Once the diagnosis of an infected necrosis is confirmed, the treatment with antibiotics, directed ideally to the isolated agents, must be instituted and some kind of intervention, in order to remove the necrotic material should be planned, since it is clear that in these cases, antibiotic therapy as a single modality is related to a low cure rate and high mortality[13]. Less clear, however, is when exactly such an intervention should be performed, since "older" necrosis tend to be more delimitated and liquefied (pancreatic abscess), allowing an easier clearance of the tissue, regardless the method chosen to remove it. Corroborating this is the observation that an early intervention on the necrosis is related to a higher mortality and a greater number of unnecessary procedures[41]. The most "traditional" method of removing the necrotic material is through an exploratory laparotomy followed by pancreatic necrosectomy, which is performed by a combination of digital dissection or delicate instrumentation, followed by debridement and washing of the peripancreatic tissue. The results of this technique have already been described in extensive series[42], and can be considered quite satisfactory, although it is clear that intervention is much more difficult and morbid when performed on a necrotic tissue that has not yet liquefied. After this procedure, the abdomen can be maintained in peritoneostomy, allowing future interventions, or suture closed and maintained in a system of continuous washing[43]. Although a more delayed intervention is desired, for the reasons exposed above, a great part of the patients with NHAP display a rapidly deteriorating clinical picture and demand an earlier surgical intervention[44].

Alternative methods to approaching the necrosis have been described, with varying grades of invasiveness. Computer tomography-guided percutaneous catheter drainage can be accomplished in cases in which tissue debridement is not necessary[45]. The video-assisted retroperitoneal approach involves the placement of laparoscopic equipment through posterior incisions, followed by the creation of a postoperative lavage system and carries the theoretical advantage of not violating the peritoneal cavity[46]. Recent literature is flourishing with case reports and small series describing the successful results of endoscopic and natural orifices surgical approaches[40, 47, 48, 49]. Also noteworthy is the approach described by Bala and cols., in which a percutaneous drainage tract is constructed initially by CT-guided puncture of the necrosis, followed by the placement of a catheter and dilation of the tract, allowing subsequent percutaneous debridement of the necrosis[5]. Regarding endoscopic/natural orifices approach, Escorrou and cols. adopted trans-gastric necrosectomy as their first line of treatment, describing therapeutic success with this single approach in 13 cases. According to them, an opening on the posterior wall of the stomach was performed through endoscopy, allowing access to the necrosis area, followed by debridement, with the additional advantage of avoiding the devastating potential complication of a pancreatocutaneous fistula[50]. More recently, a much larger series regarding the use of this technique has been published, with the results coming from multiple centers in the United States. Notably, the authors have described the resolution from the collections in 95 of the 104 patients[51]. As also previously described, endoscopic and percutaneous techniques can be combined, yielding an even higher success rate $[52,53]$. Despite very promising, it must be stressed that these techniques cannot be universally applied, still with many limitations when dealing with infected necrosis in multiple or in unfavourable sites. The need for specialized personnel and equipment is also a limitation to the widespread adoption of these techniques. More frequently, such approaches have been reserved for smaller and well delineated necrosis, located at favourable sites, such as the pancreatic tail, in stable patients[46, 54].

Although the complications of pancreatic necrosectomy have been better established for the open approach, all the techniques of pancreatic necrosectomy carry a high risk of complications (up to $62 \%$ ), such as the development of a pancreatic fistula[55], biliary stenosis, pancreatic pseudo cists, pancreatic duct stenosis with subsequent acute pancreatitis, endocrine and exocrine insufficiency[2]. NHAP 
also carries per se a high risk of pancreatic insufficiency and chronic pancreatic pain, requiring multiple hospitalizing[56].

\section{Conclusions and Future Perspectives}

SAP is still a challenge to the medical community. Consequently, many papers have been published on the subject in the later years. As a prospectus, it is noteworthy to cite the great number of experimental studies recently published evaluating therapies acting over the initial inflammatory response described in the initial phases of this disease. More consistently, the monoclonal antibody against the Tumoral Necrosis Factor Infliximab and antioxidants such as Resveratrol have shown promising results[57]. Similarly, it has been demonstrated that a great part of the etiological factors related to AP involve as a common pathway the activation of the nuclear transcription factor- $\mathrm{\kappa B}$ (NF- $\kappa \mathrm{B}$ ), a major agent in immune and inflammatory response. Following the activation of this factor, many responses such as the production of free oxygen radicals, cytokines and lymphocytes proliferation have been observed. Taking that into consideration, Leflunomide, an inhibitor of the activation of NF$\kappa \mathrm{B}$ has shown an important role in attenuating the inflammatory reactions and organic response on experimental models of AP[58]. Hyperbaric oxygen therapy was also tested in models of SAP and has shown a beneficial role in reducing the characteristic lesions caused by oxidative stress of the reactive oxygen species described in this disease[59].

From the surgical standpoint, the major perspectives are related to the improvement of the alternative modalities of approaching pancreatic necrosis. As previously described, at least five alternative modalities have been established: percutaneous debridement, which involves the assistance of imaging methods and allows a limited instrumentation of the pancreatic tissue; "traditional" endoscopic approach through the trans-gastric or trans-duodenal route, which carries the advantage of being less invasive, but has very limited indications, such as liquefied collections located at very favorable sites[60]; Natural Orifice Surgery (NOTES), using the trans-gastric route, which involves a greater incision on the posterior wall of the stomach, allowing a wider debridement of the pancreatic tissue than the one provided by the earlier endoscopic approaches[61]; laparoscopic debridement, clearly efficacious for necrosis located at sites such as para-colic gutter and the omental bursa, but only applicable in situations in which the patient is stable enough to tolerate the pneumoperitoneum[46]; and finally, retroperitoneal technique, which allows a wide access to areas not contemplated by the other techniques described above, but has the disadvantage of being more invasive[62]. These techniques are being increasingly used by many centers, which now reserve the most traditional surgical approach, that is, through laparotomy, for the cases in which these techniques fail or do not apply[5]. As a general rule, there is now a growing body of evidence favoring the treatment of infected necrosis by a "step-up" approach, in which, according to the patients response, less invasive methods are initially favored, and more invasive techniques are reserved for the non-responders[44, 63]. In accordance to that, Santvoort and cols. performed a nationwide randomized trial comparing a step-up approach, consisting in a first step of necrosis drainage, followed by video-assisted retroperitoneal lavage in non-responders, versus open necrosectomy in patients with NHAP complicated with pancreatic necrosis infection. The conclusions of this important study favored the first approach by showing it is related to a reduction in major complications such as new-onset diabetes and pancreatic insufficiency, and has also been related to an overall reduction in direct medical costs[64]. Similar results were then confirmed by the 20 year-experience described by another group[63] and have met a growing empathy from the part of many authorities. By analyzing how such issue has changed over the last few years, we can thus demonstrate how dynamic is the actual scenario concerning SAP and once again justify the need for periodic updating about this theme.

\section{REFERENCES}

[1] Frossard JL, Steer M, Pastor CM, Acute pancreatitis. Lancet, 2008. 371: pp. 143-152

[2] Amano H, Takada T, Isaji S, Takeyama Y, Hirata K, Yoshida M, Mayumi T, Yamanouchi E, et al, Therapeutic intervention and surgery of acute pancreatitis. J Hepatobiliary Pancreatic Sci, 2010. 17: p. 53-59

[3] Bradley EL \& Dexter ND, Manangement of severe acute pancreatitis. A surgical odyssey. Ann Surg, 2010. 251: p. 6-17

[4] Buchler MW, Gloor B, Müller CA, et al. Acute necrotizing pancreatitis: treatment strategy according to the stats of infection. Ann Surg 2000. 232: p. 619-626

[5] Bala M, Almogy G, Klimov A, Rivkind AI, Verstandig A, Percutaneous "stepped" drainage technique for infected pancreatic necrosis. Surg Laparosc Endosc Percutan Tech, 2009. 19: p. e113-118

[6] Bradley EL, A clinically based classification system for acute pancreatitis. Summary of the International Symposium on Acute Pancreatitis, Atlanta, Ga, September 11 through 13, 1992. Arch Surg, 1993. 128: p. 586-590

[7] Uhl W, Warshaw A, Imrie C, et al., IAP guidelines for the surgical manangement of acute pancreatitis. Pancreatology, 2002. 2: p. $565-573$

[8] Petrov MS, Revising the Atlanta classification of acute pancreatitis: Festina Lente. J Gastrointest Surg 2010. 14: p. 1474-1475

[9] Brun A, Agarwal N, Pitchumoni CS, Fluid collections in and around the pancreas in acute pancreatitis. J Clin Gastroenterol 2011. 45: p. 614-625

[10] Tsui N-c, Zhao E, Li ZL, Miao B, Cui YF, Shen YF, Qu PF, Microbiological findings in secundary infection of severe 
acute pancreatitis. A retrospective clinical study. Pancreas, 2009. 38: p. 499-502

[11] Gloor B, Muller CA, Worni M, et al., Pancreatic infection in severe pancreatitis: The role of fungus and multiresistant organisms. Arch Surg, 2001. 136: p. 592-596

[12] Kochhar R, Ahammed SKM, Chakrabarti A, Ray P, Sinha SK, Dutta U, Wig JD, Singh K, Prevalence and outcome of fungal infection in patients with severe acute pancreatitis. J Gastroenterol Hepatol, 2009. 24: p. 743-747

[13] Charbonney E \& Nathens AB, Severe acute pancreatitis: A review. Surg Infections, 2008. 9(6): p. 573-578

[14] Karimgani I, Porter KA, Langevin RE, et al., Prognostic factors in sterile pancreatic necrosis. Gastroenterology, 1992. 103: p. 1636-1640

[15] Petrov MS, Predicting the severity of acute pancreatitis: Choose the right horse before hitching the cart. Dig Dis Sci, 2011. 56: p 3402-3404

[16] Sigounas DE, Tatsioni A, Christodoulou DK, Tsianos EV, Ioannidis JPA, New prognostic markers for outcome of acute pancreatitis: overview of reporting in 184 studies. Pancreas, 2011. 40: p. 522-532

[17] Beger HG, Bittner R, Block S, et al., Bacterial contamination of pancreatic necrosis: A prospective clinical study. Gastroenterology, 1986. 91: p. 433-438

[18] Mazaki T, Ishii Y, Takayama T, Meta-analysis of prophylactic antibiotic use in acute necrotizing pancreatitis. Br J Surg, 2006. 93: p. 674-684

[19] Dellinger EP, Tellado JM, Soto NE, et al., Early antibiotic treatment for severe acute necrotizing pancreatitis: A randomized, double-blind, placebo-controlled study. Ann Surg, 2007. 245: p. 674-683

[20] Jafri NS, Mahid SS, Idstein SR, Hornung CA, Galandiuk S, Antibiotic prophylaxis is not protective in severe acute pancreatitis: a systematic review and meta-analysis. Am J Surgery, 2009. 197: p. 806-813

[21] Yao L, Huang X, Li Y, Shi R, Zhang G, Prophylactic antibiotics reduce pancreatic necrosis in acute necrotizing pancreatitis: a meta-analysis of randomized trials. Dig Dis 2010. 27: p. $442-449$

[22] Luiten EJ, Hop WC, Lange JF, et al., Controlled clinical trial of selective decontamination for the treatment of severe acute pancreatitis. Ann Surg, 1995. 222: p. 57-65

[23] Sawa H, Ueda T, Takeyama Y, et al., Treatment outcome of selective digestive decontamination and enteral nutrition in patients with severe acute pancreatitis. J Hepatobiliary Pancreat Surg, 2007. 14: p. 503-508

[24] Zavyalov T, Khotsyna Y, Tenner S, The role of antibiotics in the manangement of patients with acute necrotizing pancreatitis. Curr Infect Dis Rep 2010. 12: p. 13-18

[25] Chooklin S, Pereyaslov A, Bihalskyy I, Pathogenic role of myeloperoxidase in acute pancreatitis. Hepatobiliary Pancreat Dis Int, 2009. 8: p. 627-31

[26] Mofidi R, Suttie SA, Patil PV, Ogston S, Parks RW, The value of procalcitonin at predicting the severity of acute pancreatitis and development of infected pancreatic necrosis: Systematic review. Surgery, 2009. 146(1): p. 72-81
[27] Dambrauskas Z, Gulbinas A, Pundzius J, et al., Value of routine clinical tests in predicting the development of infected pancreatic necrosis in severe acute pancreatitis. Scand J Gastroenterol, 2007. 42:1256-1264

[28] Banks PA, Gerzof SG, Langevin RE, et al., CT-guidade aspiration of suspected pancreatic infection: bacteriology and clinical outcome. Int J Pancreatol, 2005. 18: p. 265-270

[29] Paye F, Rotman N, radier C, et al., Percutaneous aspiration for bacteriological studies in patients with necrotizing pancreatitis. Br J Surg, 1998. 85: p. 755-759

[30] Marik PE, Zaloga GP. Meta-analysis of parenteral nutrition versus enteral nutrition in patients with acute pancreatitis. BMJ, 2004. 328: p. 1407-1410

[31] Modena TJ, Cevasco BL, Basto AC, et al., Total enteral nutrition as prophylactic therapy for pancreatic necrosis infection in severe acute pancreatitis. Pancreatology, 2006. 6: p. 58-64

[32] Al Samaraee A, McCallum IJ, Coyne PE, Seymour K, Nutritional strategies in severe acute pancreatitis: A systematic review of the evidence. Surgeon, 2010. 8: p. 105-110

[33] Sun S, Yang K, He X, Tian J, Ma B, Jiang L, Probiotics in patients with severe acute pancreatitis - a meta-analysis. Langenbecks Arch Surg, 2009. 394: p. 171-177

[34] Zhang M-M, Cheng J-Q, Lu Y-R, Yi Z-H, Yang P, Wu X-T, Use of pre-, pro- and synbiotics in patients with acute pancreatitis: a meta-analysis. World J Gastroenterol 2010. 16: p. 3970-3978

[35] Winslet MC, Imray C, Neoptolemos JP, Biliary acute pancreatitis. Hepatogastroenterology, 1991; 38: p. 120-123

[36] Fan ST, Lai EC, Mok EP, et al., Early treatment of acute biliary pancreatitis by encoscopic papillotomy. N Engl J Med, 1993. 328: p. 228-232

[37] Neoptolemos JP, Carr-Locke DL, London NJ, et al., Controlled trial of urgent endoscopic retrograde cholangiopancreatography and endoscopic sphincterotomy versus conservative treatment for acute pancreatitis due to gallstones. Lancet, 1998. 2: p. 979-983

[38] Folsch UR, Nitsche R, Ludtke R, et al., Early ERCP and papillotomy compared with conservative treatment for acute biliary pancreatitis. The German Study Group on Acute Biliary Pancreatitis. N Engl J Med, 1997. 336: p. 237-242

[39] Ayub K, Imada R, Slavin J, Endoscopic retrograde cholangiopancreatography in gallstone-associated acute pancreatitis. Cochrane Database Syst Rev, 2004: CD003630

[40] Friedland S, Kaltenbach T, Sugimoto M, Soektikno R, Endoscopic necrosectomy of organized pancreatic necrosis: a currently practiced NOTES procedure. J Hepatobiliary Pancreat Surg, 2009. 16: p. 266-269

[41] Hartwig W, Maksan SM, Foitzik T, et al., Reduction in mortality with delayed surgical therapy of severe pancreatitis. J Gastrointest Surg, 2002. 6: p. 481-487

[42] Rodriguez JR, Razo AO, Targarona J, et al., Debridement and closed packing for sterile or infected necrotizing pancreatitis: insights into indications and outcomes in 167 patients. Ann Surg, 2008. 247: p. 294-249 
[43] Rau B, Bothe A, Beger HG, Surgical treatment of necrotizing pancreatitis and closed lavage: changing patient characteristics and outcome in a 19-year, single-center series. Surgery, 2005. 138: p. 28-39

[44] Besselink MGH, The "step-up approach" to infected necrotizing pancreatitis: Delay, drain, debride. Dig Liver Dis, 2011. 43: p. $421-422$

[45] Freeny PC, Hauptmann E, Althaus SJ, et al., Percutaneous CT-guided catheter drainage of infected acute necrotizing pancreatitis: techniques and results. Am J Roentgenol, 1998. 170: p. 969-965

[46] Horvath KD, Kao LS, Wherry KL, et al., A technique for laparoscopic-assisted percutaneous drainage of infected pancreatic necrosis and pancreatic abcess. Surg Endosc, 2001. 15: p. $1221-1225$

[47] Van Vyve EL, Reynaert MS, Lengele BG, et al., Retroperitoneal laparostomy: a surgical treatment of pancreatic abcesses after an acute necrotizing pancreatitis. Surgery, 1992. 111: p. $369-375$

[48] Seifert H, Wehrmann T, Schmitt T, et al., Retroperitoneal endoscopic debridement for infected pancreatic necrosis. Lancet, 2000. 356: p. 653-655

[49] Papachristou GI, Takahashi N, Chahal P, Sarr MG, Baron TH, Peroral endoscopic drainage/debridement of walled-off pancreatic necrosis. Ann Surg, 2007. 245: p. 943-951

[50] Escorrou J, Shehab H, Buscail L, Bournet B, Andrau P, Fourtanier G, Peroral transgastric/Transduodenal necrosectomy. Success in the treatment of infected pancreatic necrosis. Ann Surg, 2008. 248: p. 1074-1080

[51] Gardner TB, Coelho-Prabhu N, Gordon SR, Gelrud A, Maple JT, Papachristou GI, Freeman ML, Topazian MD, Attam R, Mackenzie TA, Baron TH, Direct endoscopic necrosectomy for the treatment of walled-off pancreatic necrosis: results from a multicenter U.S. series. Gastrointest Endosc, 2011. 73: p. $718-726$

[52] Ross A, Gluck M, Irani S, Hauptmann E, Fotoohi M, Siegal J, Robinson D, Crane R, Kozarek R, Combined endoscopic and percutaneous drainage of organized pancreatic necrosis. Gastrointest Endosc, 2010. 71: p. 79-84

[53] Gluck M, Ross A, Irani S, Lin O, Hauptmann E, Siegal J, Fotoohi M, Crane R, Robinson D, Kozarek RA, Endoscopic and percutaneous drainage of symptomatic necrosis reduces hospital stay and radiographic resources. Clin Gastroenterol Hepatol, 2010. 8: p.1083-1088
[54] Connor S, Ghaneh P, Raraty M, et al., Minimally invasive retroperitoneal pancreatic necrosectomy. Dig Surg, 2003. 20: p. $270-277$

[55] Fotoohi M, D'Agostino HB, Wollman B, et al. Persistent pancreatocutaneous fistula after percutaneous drainage of pancreatic fluid collections: role of cause and severity of pancreatitis. Radiology, 1999. 213: p. 573-578

[56] Gupta R, Wig JD, Bhasin DK, Singh P, Suri S, Kang M, Rana SS, Rana S, Severe acute pancreatitis: The life after. J Gastrointest Surg, 2009. 13: p. 1328-1336

[57] Fantini L, Tomassetti P, Pezzilli R, Manangement of acute pancreatitis: current knowledge and future perspectives. World J Emerg Surg, 2006. 23: p. 1-16

[58] Kutluana U, Oruc N, Nart D, Kaptanoglu B, Yonetci N, Ozutemiz O, Leflunomide. Is a new oral agent in treatment of acute pancreatitis? Pancreas, 2010. 39(2): p. 237-242

[59] Uysal B, Yasar M, Ersoz N, Coskun O, Kilic A, Cayc T, Kurt B, Oter S, Korkmaz A, Guven A, Efficacy of hyperbaric oxygen therapy and medical ozone therapy in experimental acute necrotizing pancreatitis. Pancreas, 2010. 39: p. 9-15

[60] Fogel EL, Endoscopic Pancreatic Necrosectomy. J Gastrointest Surg, 2011. 15: p.1098-1100

[61] Isayama H, Yamamoto K, Mizuno S, Yashima Y, Togawa O, Kogure H, Sasaki T, Sasahira N, Nakai Y, Hirano K, Tsujino T, Tada M, Kawabe T, Omata M, NOTES and endoscopic pancreatic necrosectomy for the GI endoscopist. J Hepatobiliary Pancreat Surg, 2009. 16: p. 270-273

[62] Navaneethan E, Vege SS, Chari ST, Baron TH, Minimally invasive techniques in pancreatic necrosis. Pancreas, 2009. 38(8): p. $867-875$

[63] Zerem E, Imamovic G, Susic A, Hacacic B, Step-up approach to infected necrotising pancreatitis: a 20 -year experience of percutaneous drainage in a single centre. Dig Liver Dis, 2011. 43: p. $478-483$

[64] Santvoort HC, Besselink MG, Bakker OJ, Hofjer HS, Boermeester MA, Dejong CH, van Goor H, Schaapherder AF, van Eijck CH, Bollen TL, van Ramshorst B, Nieuwenhuijs VB, Timmer R, Laméris JS, Kruyt PM, Manusama ER, van der Harst E, van der Schelling GP, Karsten T, Hesselink EJ, van Laarhoven CJ, Rosman C, Bosscha K, de Wit RJ, Houdijk AP, van Leeuwen MS, Buskens E, Gooszen HG, A step-up approach or open necrosectomy for necrotizing pancreatitis. $\mathrm{N}$ Engl J Med, 2010. 363: p. 1491-1502 